\title{
استيراتيجيات العلاقات العامة في إدارة الازمة الصحية
}

\section{دراسة تحليلية لخطابات خلية ازمة كورنا في واسط}

\author{
م. م مرائد عبد الكاظم الوائلي \\ كلية الاداب / قسم الاعلام
}

تاريخ الاستلام : 241-01-24

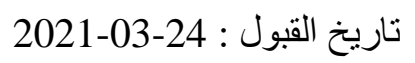

المستخلص:

تثبت إدارة العلاقات العامة أنها وظيفة اتصالية وادارية فعالة من خلال أدواتها الأصلية والفعالة في مواجهة التحديات الحالية للمنظمة والمجتمع ، بغض النظر عن النشاط التي ترتبط بها؛ اذ يمكنها دعم حل المشكلات وحالات الأزمات ، وتعزيز سمعة ملات الحكومة أو المنظمة ومنتجاتها أو خدماتها في حالات الأزمات، فيكون عمل ادارة استراتيجيات العلاقات العامة مطلق وإلزامي

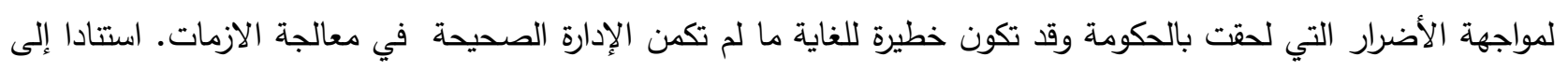
هذه الاعتبارات ، فإن الغرض من الورقية البحثية هي: تقديم مفهوم استراتيجيات اصلاح الصورة لدى ادارة العلاقات العامة ودورها الأساسي في اصلاح صورة الحكومة في الأزمات، ولتحليل وتقييم عمل العلاقات وتحليل كيفية إدارة حالة الأزمة والإجراءات المتخذة في هذا الصدد وتأثيرها في نتائج النهائية لعمل الحكومة.

أن أستراتيجيات اصلاح الصورة في العلاقات العامة قد تدعم الحكومات في التتبؤ والرد بشكل مناسب في حالات الأزمات ، إذا تم تطبيقها وفقًا للأسس العلمية الصحيحة، فأن التواصل الجيد أثناء الأزمة له القدرة على تحسين فكرة الجمهور وإيقاف ردود الفعل السلبية اتجاه الحكومة.

الكلمات المفتاحية : استراتيجات العلاقات العامة ، العلاقات العامة في الازمات ، اصلاح الصورة . 


\title{
Public Relation Strategies in Health Crisis Management Analytical Study of Wasit Crisis cell speeches of Covid-19
}

\section{Receipt date: 2021-01-24}

\section{Date of acceptance: 2021-03-24}

\begin{abstract}
Public Relations management proves to be an effective communication and management function through its original and effective tools in facing the current challenges of the organization and society, regardless of the activity it is associated with; As it can support solving problems and crisis situations, and enhance the reputation of the government or organization and its products or services in crisis situations, so the work of managing public relations strategies is absolute and mandatory to face the damage caused to the government, and it may be very dangerous unless the correct administration is in handling the crises. Based on these considerations, the purpose of the research paper is: to present the concept of image-reform strategies for the Public Relations Department and its primary role in reforming the government's image in crises, and to analyze and evaluate the work of relations and analyze how to manage the crisis situation and the measures taken in this regard and their impact on the final results of the government's work.

Image reform strategies in public relations may support governments in predicting and responding appropriately in crisis situations, if applied according to the correct scientific foundations, good communication during a crisis has the ability to improve the public's perception and stop the negative reactions towards the government.
\end{abstract}

Keywords: public relations strategies, public relations in crises, image repair. 
لا يمكن للمؤسسات الحديثة أن تعمل في المجتمع الحالي دون مساهمة فعالة من قبل إدارة العلاقات العامة في التواصل والاستراتيجيات والبرامج التي تطورها ، هذه تسهل إنشاء اتصالات قائمة على الثقة بين الحكومات وفئات عديدة من الجماهير، وبناء علاقات تفاعلية بين مختلف مستويات المجتمع لإنثاء مناخ من الثقة والتعاطف بين موظفيها، وكذلك بين الجمهور . تذكر ادبيات الوظيفة حقيقة أن ادارة العلاقات العامة تمثل وظيفة إدارة متميزة، والتي تدعم بناء والحفاظ على التواصل، والتعاون بين الحكومة والجمهور، يعني إدارة المشاكل أو النزاعات، ويحدد ويؤكد مسؤولية الإدارة لخدة الجمهور وبالتالي، فإن الوظيفة الإدارية تدعم بناء العلاقات المفيدة بين الحكومة وجمهورها ، بين المنظمة والبيئة ، عن طريق الاتصال المتماثل. والأنشطة الرئيسة المميزة للعلاقات العامة تهدف إلى دراسة الرأي العام ، والموقف والجوانب التي يمكن أن تؤثر، للأفضل أو للأسوأ على أعمال وخطط المنظمة، بحيث يكون الجمهور على اطلاع وقادر على الفهم لهذا فإن ممارسة استراتيجيات ادارة العلاقات العامة يجب أن تعني بالاحتراف والمسؤولية الاجتماعية والاخلاقية في تبني سياسات واتجاهات العمل ، بحيث تتزامن مصلحة الحكومة مع مصلحة الجمهور والا فان التحيزات لطرف معين يكون خطير للغاية إذا لم يتم إدارة وضع الأزمة بثكل صحيح وذلك للحد من الآثار السلبية أن التواصل الجيد أثثاء الأزمة لا يمكن أن يكون إلا مفيدًا وقادرًا على التحسن بل ويتوقف ردود الفعل السلبية للجمهور ، وبالتالي تصبح عملية الاتصال علامة مرجعية يكون الغرض الرئيسي منها بناء استراتيجية اصلاح صورة الحكومة في الازمات تتعلق بمتطلبات الاتصال من منظور كل جمهور ، لأن النهج الذي يقوم على الجمهور يشجع المنظمة على تحليل آلية

الاتصال الخاصة بها.

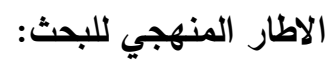

أولا : مشكلة البحث :

قد تتأثر صورة المنظمة بشكل خطير في حالة الأزمات ، وتكون ثقة الجمهور بها تقلصت إلى حد كبير ، وفقا لحجم ومدة الأزمة متسببة حالة من الذعر والارتباك بين الجمهور والحكومة ـ هنا يمكن لإدارة العلاقات العامة الحكومية بالفعل أن تشارك في بناء واعادة وترميم الصورة المتضررة للحكومة من خلال مد جسور التعاون مع الجمهور ومساعدة الحكومة على ممارسة التواصل الفعال مع الجمهور عن طريق انشطتها بعدها أداة انموذجية وليس تكتيكية ، وتكمن مشكلة بحثنا في الكثف عن أستراتيجيات إصلاح صورة الحكومة لاى إدارة العلاقات العامة في الأزمات دراسة تحليلية لخطابات خلية ازمة كورنا في محافظة 
هناك علاقة بين ممارسة أستراتيجيات الإتصال في إدارة العلاقات العامة والحفاظ على الصورة الإيجابية للحكومة أثناء الأزمات

ولاسيما في الازمات الصحية .

ثانياً :اهداف البحث : (البي

تهدف هذه الورقة البحثية إلى تحليل الطريقة التي دارت بها الحكومة حالة ازمة كورونا اتصالياً واثر استراتيجيات العلاقات العامة في رسم صورتها امام الجمهور والكثف عن الانشطة الاعلامية التي أجريت في هذا الصدد مع الكثف عن الوسائل الاتصالية

المعتمدة ، ومعرفة مدى تأثر استراتيجيات اصلاح صورة الحكومة لدى ادارة العلاقات العامة اثناء الازمات الصحية . 1. معرفة مدى تطابق نظرية أستراتيجيات إصلاح صورة الحكومة لاى إدارة العلاقات العامة في الأزمات الصحية ولاسيما

$$
\text { في خطابات خلية الازمة في محافظة واسط . }
$$

2. التعرف على معرفة الآثار التي يمكن أن يستخلصها ممارسو العلاقات العامة من دراسة الازمات الصحية.

3. معرفة الأنشطة الاتصالية في العلااقت العامة لإدارة الازمة الصحية ومدى فاعليتها في التاثير على الجمهور.

ثالثآ: أهمية البحث:

اذ تكمن أهمية مراجعة استراتيجيات اصلاح الصورة في ادارة العلاقات العامة اثناء الازمات ومدى حقيقة أنها تسلط الضوء على كل من أمثلة إيجابية وسلبية فيما يتعلق بإدارة العلاقات العامة في الازمات التي تعصف بالحكومة المحلية اثناء مواجهة تلك

$$
\text { الازمات في الجانب الصحي . }
$$

رابعآ: تساؤلات البحث

1- ماهي أستراتيجيات إصلاح صورة الحكومة لدى إدارة العلاقات العامة في الأزمات الصحية؟

2- ما هي الآثار التي يمكن أن يستخلصها ممارسو العلاقات العامة من دراسة الازمات الصحية ؟

3- الكثف عن الأنشطة الاتصالية في العلاقت العامة لإدارة الازمة الصحية ومدى فاعليتها في التاثير على

الجمهور؟

خامسآ: منهج البحث:

أستخدمت الدراسة المنهج المسحي واعتمد الباحث اداة تحليل المضمون بوصفها احد أدوات المنهج المسحي كونه يمثل المنهج المناسب للوصول الى اهداف البحث وهي- تحليل المضمون- كأداة لجمع وتحليل بيانات خلية الازمة الخاصة بوباء كورونا للتوصل إلى الوصف الكمي والمحتوى للرسالة الإعلامية، وقد تم إختيار وحدة الموضوع أو الفكرة، إذ تُعدّ هذه الوحدة أكثر وحدات تحليل المحتوى شيوعا، لان تتاولها يفيد في: تحديد أكثر الفئات استخداما في الكثف عما يقوله المحتوى، كما تم اختيار وحدة الكلمة لمعرفة كيفية قيام الحكومة برسم واصلاح صورتها اثناء وبعد الازمات ، اما بخصوص فئات التحليل فقد تضمن التحليل فئتي ؛ الموضوع ماذا قيل ؟ وفئة الثكل كيف قيل؟ (حسين، 1999). 


\section{سادسا: أدوات البحث :}

اعتمد الباحث في دراسة مشكلة البحث أداة تحليل المضمون ضمن أدوات المنهج المسحي وذلك لرؤية الباحث بالوصول الى النتائج خلال تحليل الخطابات التي صدرت عن خلية الازمة في المحافظة بالنسب المئوية وذلك تحديد الالولوية في تطبيق

استراتيجيات اصلاح الصورة .

\section{عينة البحث والمدة الزمنية للتحليل:}

تم تحليل مضمون جميع خطابات وقررات خلية الازمة في محافظة واسط ، وقد بلغ مجموع الأعداد التي خضعت للتحليل، والخاصة بـ(بخطابات خلية الازمة في محافظة واسط) التي تثكلت وفق الامر الديواني رقم (55) لسنة 2020 الصادر من الأمانة العامة لمجلس الوزراء واستتادا للامر الإداري المرقم بالعدد 24/5/1 في 2020/2/20 الصادر من محافظة واسط /مكتب المحافظ المتضمن تثكيل لجنة عليا لغرض متابعة إجراءات السيطرة على انتشار فايروس كورونا بواقع ( 27 ) خطاباً، من بداية تفشي ظهور الوباء وظهور الازمة الصحية وتثكيل الامر الديواني الخاص بخلية الازمة وبالتحديد من 2020/2/17

$$
\begin{aligned}
& \text { لغاية 2020/3/28 . } \\
& \text { الاطار النظري للبحث : } \\
& \text { أولا : الأزمات الصحية : } \\
& \text { 1- مفهوم الازمة : }
\end{aligned}
$$

ان تعدد المفاهيم والمصطلحات جعلتها - المفاهيم والمصطلحات - تمتاز بالغموض ويمكن تعريف الازمة على انها حالة غير مستقرة ينتج عنها عوامل مؤثرة في وقت محدد مما يترتب عليه اتخاذ قرارات طارئه وبفترة زمنية قصيرة (عبد العزيز بن سلطان الضويحي، 2004 ص30). " الا ان وجهة نظر مماثلة عقدت من قبل (تييري سي) الذي يعرف الأزمة بانها "اضطراب يؤثر جسديًا على النظام كله ويهدد افتراضاته الأساسية ، والثعور الذاتي لووجودها؛وهم بذلك يصفون ثلاثة آثار وجودية للأزمة هي

(Petrovici, 2014) :

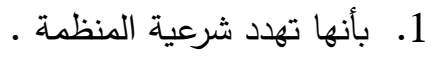 2. تعكس المهمة الاستراتيجية للمنظمة. 3. تغير الطريقة التي يرى بها الجهور أنفسهم.}

ويمكن تعريف مفهوم الأزمة ؛ على انها حالة من الثذوذ الذي يسببه على المستوى الفردي ، وكذلك في حياة المنظمة أو المؤسسة، وهي خلل غير متوقع وموقف مفاجى نتيجة اوضوع غير مستقرة ينتج عنها اتخاذ قرارات اثناء تلك المواقف (هدى عبد الرضا الجميلي ، 2018 ) كما ان المعاني المختلفة المعطاة لهذا المصطلح والتي قدمها الأدب تصفها بأنها مجموعة من المشكلات الداخلية والخارجية التي تهدد سلامة أو سمعة أو وجود فرد أو منظمة حالة غير سارة تؤثر بشكل خطير على عمليات المنظم تعد الأزمات بمثابة حالات البادئة (تحذير مسبق) التي تتعرض لخطر تتصاعد في شدتها ، وتتدرج تحت وسائل 
الإعلام الوثيقة أو التدقيق الحكومي ، بين التعامل مع العمليات العادية ، وتعريض الصورة التظظية للخطر بيت القصيد للشركة "الأزمة هي حدث كبير لا يمكن التتبؤ به والذي قد يكون سلبيا النتائج، قد يؤدي الحدث وما بعده إلى إلحاق ضرر كبير بالمنظمة وموظفيها ، والمنتجات ، والخدمات ، والوضع المالي ، والسمعة " كما أن هناك تتوع في المصطلحات المستخدمة لوصف المراحل المختلفة للأزمة ، لكن هناك اتفاق عام على هيكلها ومراحلها "دورة حياة الكوارث" وتتكون من أربع مراحل متميزة

(Guth, 1995): هي

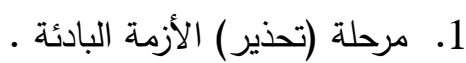

2. مرحلة الأزمة (يتم الوصول إلى نقطة اللاعودة ، ويتم بعض الضرر ) .

3. مرحلة المزمنة (فترة مباشرة).

4. ومرحلة حل الأزمة والمعالجة المؤقتة يتم اتخاذ تدابير مؤقتة لاعم الوظائف الأساسية ومن ثم عودة الأشياء إلى وضعها

• الطبيعي

وقد تكون الازمة الصحية تتميز بحالة غير سارة تؤثر بشكل خطير على عدد من العوامل التي تؤثر جسديا على أداء النظام الصحي والتتظيمي بأكمله وتهديد هويته وسبب وجودها ؛ اي عملية ذات تأثير خطير يهدد سلامة المنظمة غير المخطط لها وعملية غير مرغوب فيها قد تعرض للخطر بشكل كبير على وجود المنظمة وفي المدى الطويل بأكملها مما يؤدي للحدث الذي يحدث تحت ضغط الوقت وفيه إجابات سريعة ضرورية ويتم استيعاب الأزمة لتلك الظواهر التي تثني الفرد ، وكذلك المنظمة ، ويتم تحديدها في المقام الأول من خلال مدة عدم القدرة على اتخاذ قرار ، الفاصل الزمني الذي يتم خلاله إزعاج عمل المنظمة ، (Sherry Jean Holladay, 2013). (أحيانًا يتعرض للخطر

\section{2- 2 إدارة العلاقات العامة في الازمات الصحية :}

لايوجد تعريف محدد وشامل لمفهوم العلاقات العامة يحصر ذلك المفهوم اذ انها تتعدى وظائفها ولاسيما في المجتمعات الحديثة ولاسيما ان مفهومها يختلف بحسب المجال التي تعمل به ولكن يمكن القول ان مفهومها ينحصر بين المؤسسة وبين جمهورها بغض النظر عن مجال الممارس لها ، اذ تعرف بانها نشاط اتصالي مخطط له يهدف لكسب الجمهور المحدد والمستهدف وباستخدام وسائل الاتصال المختلفة (علي عجوه، 2003 ص24) ، لذلك ان العلاقات العامة تهتم بكسب الجمهور باستخدام كافة الوسائل الاتصالية وهي وظيفة إدارية وإعلامية قادرة على التخطيط والتتسيق والمتابعة ووضع البرامج الكفيلة بتحقيق الأهداف . توجد علاقة بين وظيفة العلاقات العامة والادارة التظيمية خلال تجربة الأزمات وهذه العلاقة ملحوظة في اغلب المؤسسات العراقية خاصة عند غياب خطير للتخطيط للأزمات والتدريب في المنظمات، اذ تضطلع العلاقات العامة بأدوار مختلفة في المنظمات المختلفة ، بدءا من فني لمدير، أي دور اداري في الأزمات والمنظمات الحالية بالرغم من التحديات المالية والاجتماعية والأخلاقية الهامة، ويحدد بعض الباحثين ادواراً بناءً على ممارسة العلاقات العامة داخل هيكل الإدارة التنظيمية 
للمؤسسة ، بينما يحدد الآخرون هذه الأدوار بناءً على أدوار ممارس العلاقات العامة من حيث الاتصالات، بينما ركزوا باحثين اخرين على البيئة التي يعمل فيها ممارسو العلاقات العامة :(Rhee1, 2014) ، ان ميدان الصحي في العراق ولاسيما اثثاء حدوث الازمات يكون من اولياته تجاوز تلك الازمات ، وهذا يقع على عاتق إدارة العلاقات العامة وخاصة بمجال انتشاء الأوبئة والامراض الانتقالية ،يوفر تصميم هذه الورقة البحثية فرصة لاستكثاف حجم المؤسة والدور التي تلعبه العلاقات العامة داخل المنظمات الصحية في الأزمات، على الرغم من أن جميع المنظمات تواجه خطرًا محتملًا ، حتى ان أكبر المنظمات هي أكثر عرضة للخطر ولازالت المنظمات الكبيرة عرضة للأزمات اكثر من المنظمات الصغيرة.

\section{3- إدارة العلاقات العامة وادوارها للازمات الصحية :}

ان إدارة العلاقات العامة للازمات الصحية تلعب دور أساسي ومهم تحقيق دور مهم لتوفر هذه الإمكانات ، وبدورها تؤدي إلى الحصول على سيطرة معينة على السلوك الأزمة ، وقد تستشعر العلاقات العامة الفعالة في منع العديد من حالات الأزمات المحتملة، إذا تسهم في مراقبة البيئة الخارجية والداخلية وتثير إلى حقيقة السياسة أو الممارسة الضارة بالمؤسسة . Bourne, (2019، تأتي الأزمات بكافة أنواعها وتتفاوت درجاتها خطورتها في مجال العلاقات العامة وقد تم تأكيد خطة الأزمات المعتمدة من قبل المتخصصين والأكاديميين على حد سواء أي أن المنظمة يمكن تتوقع الأزمات التي قد تحدث وبالتالي تخطط للتعامل السريع معها مثل هذه الحالات بمجرد وضع الخطة ، يجب اختبارها ، غالبًا من خلال محاكاة تدرببات الأزمات أو من خلال مناقثات منتظمة مع الإدارة لمراجعتها إجراءات الاستجابة للأزمات ويرى (أميس)؛ ان نجاح معالجة اللازمة يتحدد في اربع خطوات حاسمة على المديرين اتباعها هي : (L.Benoit, 2006) ) أ- القيادة المبكرة والكفؤة ، والتي تتضمن القرار اتخاذ إجراءات فورية من قبل كبار التتفيذيين. ب- - مقاربة ذات أولوية ،والتي تتضمن إدارة سريعة ومشرفة للمشكلة الفعلية ، على الفور والتواصل مع الأشخاص الأكثر تأثرا بثكل مباشر ، ومعالجة الضحايا الاحتياجات .

ت- - الحفاظ على السمعة واستراتيجيات الانتعاش ، التي تتطوي على سرعة العمل ، والقرارات السليمة في الأساس ، وإبلاغ المعلومات المفيدة أولئك الذين يحتاجون إليها (قد لا يكون هذا بالضرورة وسائل الإعلام) . ث- التخطيط الناجح : والذي يثمل على مجموعة امور من الاجراءات هي : • التفويض المسبق الذي تستطيع المنظمة من خلاله التصرف الفوري فقط وناجحة من تلقاء نفسها ، مع القليل من

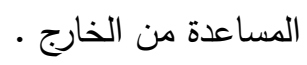
• استجابة أولى قاطعة تتضمن الانفتاح والاستجابة وحقيقة الحقيقة التعاطف مع الكثف الفوري.

وهنا يتم تحديد ادوار ممارسة العلاقات العامة في الازمات الصحية بثكل منظم مما يثكل حالة من الوضوح على العكس عدم تحديد تلك الأدوار سيعرض إدارة العلاقات العامة للازمة الصحية للغموض وهنا حدد المعلم (ريكس هارلو) حوالي500 تعريف 
مختلف حول مفهوم العلاقات العامة وكذلك الممارس الهولندي فرانس فورهوف، اذ اختلف كلهم في تحديد ادوار العلاقات العامة (Anna Oksiutycza, 2011): في اثثاء الازمات لكن هنالك اتفاق لاى الباحثين الاخرين في تحديد ادوالاها كالاتي أ- دور اداري والمتعلق ببناء استراتيجيات العلاقات العامة بين المنظمة والجماهير •

$$
\text { ب- - دور عملي في اعداد الرسائل الاتصالية وصياغتها . . }
$$

ت- دور يتعلق بالتنسيق بين قيم المجتمع واتجاهات المؤسسة . ث- - دور تعليمي بناء سلوكيات جديدة لأعضاء المنظمة في الداخل والخارج.

ثانيا : دور وتوظيف الأنثطة الاتصالية للعلاقات العامة اثناء إدارة الازمات الصحية : تعرف الأنشطة الاتصالية بأنها اختيار وسائل الاتصال في ادارة العلاقات العامة اثناء الازمات بنقل رسائلها الاتصالية ،اذ تكون كل وسيلة تساعد في استهاف فئات محددة من الجمهور ، ولابد أن توظف وسائل الاتصال بشكل صحيح وأن مقومات نجاح إدارة العلاقات العامة تعتمد على استثمار تلك الوسائل الاتصالية بشكل فعال (عبد النبي عبد الله الطيب ، 2016 ص35).

\section{1}

تعد الوسائل الاتصالية التي تعتمد على شبكات الانترنت بكافة مسمياتها من الوسائل المؤثرة والسريعة في نقل الرسائل إلى الجمهور المستهدف ، إذ أصبحت تلك الوسائل أسهل من حيث الاستخدام وغير مكلفة مع إمكانية استثمار الوقت وسرعة النقل وهذا النوع من الرسائل يندرج تحت مسمى(Electronicmessages)الرسائل الالكترونية (محمد الباوي ، 2004 ص35) ، واصبحت الرسائل الموجهة إلى الجمهور تحمل الكثير من المفاهيم والمضامين وبشكل مباشر (كريم الجاف ، 2013 ص130)

\section{: - : - مبكات التواصل الاجتماعي -}

تعد شبكات التواصل الاجتماعي من الوسائل التي تساعد على تحقيق التفاعلية بين المرسل والمتلقي بصورة مباشرة ، مما يسمح بدراسة رجع الصدى للرسالة بشكل آني وسريع ، مع تحقيق الإقناع للجمهور (كاظم عمران موسى ،صفد حسام الساموك ، الطبعة الثانية 2013 ص23). ومن أهم هذه التطبيقات هي:

\section{: Facebook : الفيس بولك}

هو واحد من أهم شبكات التواصل الاجتماعي في العالم الذي تم إنشائه في مدينة فيفري عام 2004 الذي قام بتصميمه (مارك زوكريرغ) (*) ، وأصبح هذا الموقع واحدآ من أهم مواقع التأثير بالمجتمعات (احمد القاسمي وسليم جداري ، 2019 ص21).

:Twitter : تويتر

- مارك زوكربيرغ، 14 مايو 1984، رجل أعمال ومبرمج أميركي، ولد في وايت بلينس، نيويورك، الولايات المتحدة الأمريكية.

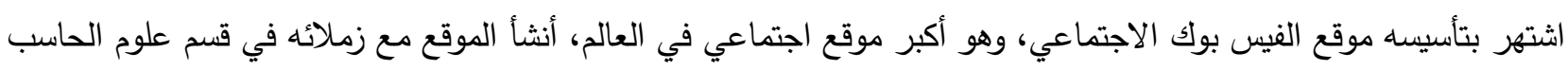
داستن موسكوفيتز وكريس هيوز وهو في جامعة هارفارد. وهو بمثابة الرئيس التتفيذي لموقع الفيسبوك. 
لا يختلف كثيرا عن الفيس بوك من حيث التأثير والانتقاء للجهور ، وإيصال الرسالة للجهور ، وهو موقع صمم في يناير 2006 لصالح شركة Obvious الأمريكية في مدينة سان فرنسيسكو من (إيفان ويليامز)(*) .

\section{2. الوسائل الاتصالية والإعلامية :}

تعد وسائل الاتصال الإعلامية ذات تأثير في عملية نقل الرسائل الاتصالية بين القائم بالاتصال والجمهور، ، لأن ذلك يساعد على انتقاء الوسائل الإعلامية التي تساعد في الاتصال مع الجمهور المستهدف ومن أهم تلك الوسائل بصورة عامة : - التلفزيون:

وهو من وسائل الاتصال المهمة والأكثر شعبية لدى الجمهور ،على الرغم من التطور الحاصل بالأجهزة الآكترونية الأخرى وحتى التلفزيون أصبح يتمتع بتقنيات هائلة من البرامج والتحديثات واختيار البث التلفزيوني (عبد الله تايه ، 2006 ص33) . -

هو من الأجهزة المهمة جدا والقريبة لدى الجمهور ، وهو جهاز يعتمد على ترددات البث اللاسلكي وهو بمتاول جميع المستخدمين -

تعد الصحيفة من وسائل الإعلام التي لها شعبية كبيرة في المجتمعات ، ورغم التطور التكنلوجي وتتوع وسائل الاعلام الأكثر تقدما الا أنها تمتعت بمساحات واسعة من الأخبار والأحداث اليومية . - المطويات والفلودرات التعريفية والبوسترات .

هي عبارة عن مطويات ورقية تحمل الرسوم التوضيحية والرسائل القصيرة والإرشادات والتوجيهات بشكل واضح ، حسب طبيعة الحملة وهي تتمتع بسهولة إيصال المعلومة (انتصار ابراهيم عبد الرزاق ، صفد حسام الساموك ، 2011 ص27). لذلك يمكن توظيف تلك الوسائل ضمن أنشطتها الاتصالية في ادارتها للازمة الصحية خلال توزيع مهام هذه الوسائل بصورة منظمة مما يثكل حالة من التجانس في إيصال رسائلها الموجة للجمهور ان تحديد وظيفة المتحدث الرسمي باسم المنظمة خلا الاستجابة للأزمة بسبب قدرتها الفريدة على نشر المعلومات على الفور تقريبًا (Natalie A. Brown, , 2013) تشكل حالة من المتابعة والجذب من قبل الجمهور وتظهر هذه النتيجة جلية في اغلب الدراسات تشير الى أهمية وسائل التواصل الاجتماعي في الاستجابة للأزمات لكون وظيفة العلاقات العامة الاتصالية تكافح من اجل بقاء هوية المنظمة عن طريق الاتصال المستمر مع الجمهور ويحقق الاتصال في العلاقات العامة اثثاء الازمات مجموعة من الاجراءات ابرزها: Peggy Simcic Brønn And)

Erik L. Olson, 1999,)

- إيفان ويليامز ، واسمه الكامل: إيفان كلارك ويليامز، وهو رجل ريادة أعمال أمريكي، من مواليد 31 مارس 1972م، وكان

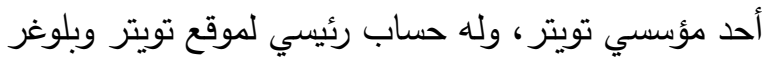


أ- هنالك اتفاق عام على دور الاتصالات كمساهم في تحقيق استراتيجية العلاقات العامة داخل المنظمات أو يجب أن تكون ذات أهمية متزايدة.

ب- - في الواقع ، الاستخدام "الاستراتيجي" للاتصالات هي الخيط المشترك الذي يمر عبر وظيفة العلاقات العامة. ت- بعد النظر في العديد من التعريفات المختلفة للاستراتيجية ، قرارات استراتيجية مكتوبة مثل تلك التي هي غير منظمة وغير روتينية ، أي فريدة من نوعها وتستخدم قواعد قرار معقدة ، ذات أهمية خاصة بالنسبة للمنظمات -وتكون بشكل عام معقدة للغاية. يقول كوين إن القرارات الاستراتيجية تردع او تقلل من وقوع الازمات . ث- تلغى الاتجاه العام للمنظمة وقدرتها النهائية على البقاء في كثير من الأحيان في بيئة تتميز بما يمكن التتبؤ به ، وغير متوقع ، وتغييرات غير معروفة. يبقى السؤال ، مع ذلك ، ما إذا كانت العلاقات العامة تم تجهيز المديرين لتقديم المشورة الاستراتيجية للإدارة العليا في مثل هذه بيئة. احياناً قد تفشل الاتصالات اثثاء الأزمات في معالجة كافية للازمة قد يكون التقدم التكنولوجي الحديث قد غير الدور الجمهور في مثل هذه الحالات اثثاء الأزمات اذ تتطلب الأزمات استراتيجيات اتصال قوية للمنظمات للتأثير تصورات أصحاب المصلحة ، والتي يتم تعريفها على أنها " شخص أو مجموعة تتأثر أو يمكن أن تؤثر على منظمة" عندما يدرك أصحاب المصلحة أن إحدى المنظمات تواجه أزمة، يجب أن تعالج استراتيجيات الإدارة هذه المشكل اتوحل الأزمة للحفاظ على سمعة المنظمة Nمatalie A

.Brown A. C., , 2013,)

\section{استجابات الجمهور للعلاقات العامة في ادارتها للازمة الصحية :}

قد تحافظ استراتيجيات العلاقات العامة على العلاقة العامة بين المؤسسة والجمهور في جميع محاولات توظيف استراتيجية تحويلية باتباع الاستجابات العامة لمنظمة واحدة خلال أزمتها من خلال تحليل مقالات الصحف المنشورة ورسائل مواقع التواصل الاجتماعي، ويمكن تحديد أي استراتيجيات الاستجابة اذ كانت يتم توظيفها من قبل المنظمة في كل مرحلة من مراحل فترة الأزمات، ويمكن أن يوفر التحليل بعض الأفكار حول كيفية عملها إذا كان التوازن يتم بين ضرب العلاقات العامة والاعتبارات

الاخرى. (William, 1994)

وقد قست استراتيجيات الاستجابة إلى أربع فئات كان الأول يسمى التقليدي استراتيجية العلاقات العامة، والتي تضمنت نصائح العلاقات العامة التقليدية لمساعدة المؤسسة في الحصول على الصفح العام وإعادة بناء المصداقية مع الجمهور وتثمل

(William, 1994) : استراتيجيات الاستجابة في العلاقات العامة على ما يلي أ. سياسة المؤسسة بثأن القضية (إذا كان ذلك مناسبًا) . ب. التحقيق من الادعاءات التي تخص المؤسسة .

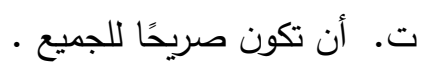
ث. الاعتراف طوعًا بوجود أزمة ، إذا كانت صحيحة. ج. الإعلان عن الإجراءات التصحيحية وتنفيذها في أسرع وقت ممكن. 
كما ان استراتيجيات الاستجابة في العلاقات العامة يمكن ان تتضمن تكتيكات الاستجابة والتي تكون بدورها قابلة للنمو والحياة

(Benait, 1997) : ويمكن النصح بها لممارسي العلاقات العامة بتوظيفها وتثمل ما يلي أ. محاولة تحويل الاتهامات بعيدًا عن انتباه وسائل الإعلام والجمهور .

ب. إعلام وسائل الإعلام بأن المنظمة هي في غضب من الموقف أثثاء اتخاذ إجراءات جوهرية ضئيلة أو معدومة . ت. أن المشكلة قد حلت أو أن مسبب الازمة يغادر المنظمة لأسباب لا علاقة لها ث. تطوير خطة اتصالات أزمة وممارستها ومعرفة من سيكون المتحدث الرئيسي ، وأن يكون منفتحًا وصادقًا مع وسائل الإعلام ، الاعتراف بكل ما تعرفه وما لا تعرفه.

ج. هنالك رؤى إضافية حول تساعد أنماط التداخل المنظمات على الاستعداد والمشاركة في الاستجابة للمشكلات، يمكن اعتماد أحد الأساليب الثلاثة:

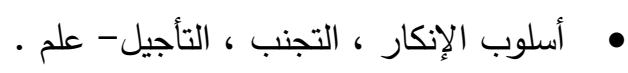

• أسلوب تكيفي ، يتميز بافتراض أن سيتم تصحيح الأخطاء في الأداء التي أدت إلى المشكلة إلى حد كبير على

$$
\text { النحو الذي تحدده القوى الخارجية . }
$$

• أسلوب ديناميكي ، والذي قد يشمل رد عدواني على النقاد الذين لا يفهمون الظروف التي أدى الى الازمة . ح. الإعلان عن التغييرات المعلقة لضمان المشكلة لا تتشأ مرة أخرى ، واتخاذ تدابير استباقية لاتخاذ القرار في الشركة لتحسين عملياتها مع المفتاح لكل أسلوب اتصال هو موضع التحكم ومتابعة رد الفعل وأسلوب الرغبة والسيطرة من جانب الأطراف المتضررة من الأزمة.

خ. الثعور بالسيطرة والتي تتضمن نتيجتها أفضل أداء وإنسان - خيارات الاتفاق التي يتعين أن تمارسها المنظمة نيابة عن جميع الأطراف معبأ بالأزمة. وبالتالي يسعى النمط الديناميكي إلى نتائج مربحة للجانبين يستخدم الأزمة كفرصة لتحقيق تحسين تعاوني، فضلاً عن أن الجمهور غالبا ما يكون أكثر انتباها خلال الأزمات ، فإن المنظمة ولديها فرصة لإثبات التزامها بالسلوك المسؤول وتحديد الخطوات التي تتخذها لتكون مسؤولة ومستجيبة لاحتياجات أصحاب المصلحة حقيقة أن الأزمات تختلف في شدتها وسببها يعني أن كل واحد يستحقها استجابة فريدة.

\section{ثالثا : نظرية إصلاح الصورة في الازمات( خطاب إصلاح الصورة) :}

النظرية مبنية على إفتراضين هما: الإتصال وهو نشاط موجه نحو الهدف والفرض الثاني هو الحفاظ على الصورة الإيجابية Williom L. للمنظمات هو الهدف الأساسي في الإتصالات اثثاء الازمات، (Joseph R. Bhrney and all, 2002) طرح 1995 نظeneit الإتصالية أثناء الأزمات أو عندما تتعرض صورة الدولة أو المنظمة للتشويه، وهنالك أختلافات في أصلاح الصور سواء للأفراد 
أو للمنظمات، فالمنظمات قد تستخدم ستراتيجيات مختلفة عن الأفراد وتوظفها في أشغال مختلفة. Benait, public) relations, review,, 1997)

تركز نظرية إصلاح الصورة على خيارات الرسالة الإتصالية بدلاً من الوقوف عند وصف أنواع الأزمات ومراحلها، بمعنى أن النظرية تعالج مايجب أن تقوم به الدولة أو المنظمة عندما تتعرض لأزمة أو عندما تكون صورتها مشوهة أو سلبية، وتقدم هذه

النظرية خمس ستراتيجيات تتطوي تحت كل ستراتيجية عدد من المتغيرات أو التكتيكات وهي كما يأتي: (Willam, 1997) . 1. الأنكار : ويندرج تحت هذه الستراتيجية متغيرين في طريقة الإنكار هما:

أ. الإنكار البسيط : ويعني إنكار حدوث الفعل ونفي ضررهُ على الدول أو المنظمات والآخرين. (Maria E. Len. Ries,

ب. تحويل اللوم : بحجة أن الدولة أو المنظمة أو شخصاً ما هم في الواقع من قاموا بهذا الفعل العدواني أو الهجومي. (Bhrney, 2002)

2. التهرب من المسؤولية: تتهرب الدول أو المنظمات أو الأفراد من مسؤوليتها أتجاه الفعل المتهمة به، وهنالك أربع أشكال لهذا التهرب هي: (Dipalma, 2011) أ. رد الفعل على الإسستفزاز : تستطيع لدولة أو المنظمة أو الفرد أن يقول أن الفعل كان مجرد رد فعل لعمل أستفزازي من الآخرين، وينظر لهذا السلوك كرد فعل معقول على هذا الإستفزاز . ب.عدم الإمكانية : تدعيي الدولة أو المنظمة أو يدعي الفرد، يدعون بنقص في المعلومات حول الموقف وعدم السيطرة والتحكم بالوضع. ج. الحادث العرضي : تزعم الدولة أن الفعل الهجومي وقع بالصدفة، وأذا كان بمقدور الدولة أن تقنع الجمهور أن الفعل وقع عن طريق الخطأ، وهنا يجبب أن تقل المسؤولية، وينبغي خفض الأضرار التي لحقت بصورة الدولة أو المنظمة أو الفرد. د. النوايا الحسنة : تعني أن الدولة أو المنظمة أو الفرد عملت بسلوك( الفعل) بنوايا حسنة، ولم يكن قصدها سيئاً. 3. تخفيف شناعة الحدث: الدولة التي تتهم بالأفعال الخاطئة يمكنها لأن تقلل من درجة شناعة الحدث لإصلاح صورتها ويمكن أن تتبع التكنيكات التالية: (L.Benoit, 2006) أ. التعزيز والتفاخر: تستخدم الدولة صيغة التعزيز لدعم المشاعر الإيجابية للجمهور إتجاه الدولة، للتعويض عن المشاعر السلبية المرتبطة بالفعل الخاطئ، بالفعل تصف خصائصها وأفعالها الإيجابية الماضية وتاريخها العريق. ب. تقليل شناعة الحدث : ويعني تقليل المشاعر السلبية المرتبطة بالفعل الخاطئ لتقليل الضرر على الدولة. ج. التفاضل: تستخدم الدولة التفاضل عندما تصنف الفعل ضمن أعمال أُخرى مماثلة ولكنها أكثر سخطاً وشناعة. د. التفوق : تستخدم التفوق لتخفيض درجة الإساءة أو للحد منها، ووضع الفعل في سياق مناسب وإيجابي. وتدعي أن الفعل الحالي أقل خطورة. 
هـ. الهجوم المضاد : فالدولة المتهمة بسوءعمل قد تهاجم بتهم لإثارة الثك لدى الجمهور عن مصداقية التهمة مابعدها عن الدولة لإصلاح الصورة التي قد تتكرر عنها.

و.التعويض : هو الثكل النهائي للحدمن وجهة الإساءة، أذا كان مقبولاً من الضحية وينبغي تحسين صورة الدولة عن طريق تقديم تعويض للمتضررين.

4. الإطارات التصحيحية: وهي إحدى الستراتيجيات العامة لإصلاح الصورة، وهي بيانات تعرف الدولة عن إلتزامها بإصلاح الضرر الناجم عن الفعل، ويمكن أن يأخذ هذا الإجراء لإصلاح إستعادة الوضع الموجود قبل الفعل الذي أثار المشكلة، والوعد بعدم تكراره، توصل باحثين إلى أن شركة( Bp) النفطية كانت تصف ماكانت الشركة تعملهُ من إجراءات للسيطرة على التسريب النفطي في خليج المكسيك أي أنها استخدمت ستراتيجية الإجراءات التصحيحية ومن ثم تعويض الضحايا، في الوقت نفسهُ لم تشغل ضمن ستراتيجياتها تحويل اللوم إلى الشركات الأخرى المشتركة بالكاثة أيضاً. (Harlow, 2011) 5. الإعتراف بالذنب( الإعتار): وهي الستراتيجية الأخيرة لإصلاح الصورة وبناءها، وتعني الإعتراف بالخطأ وطلب الصفح والإعتذار للجمهور، العيب المحتمل لهذه الستراتيجية هو أنه قد تثير دعاوي قضائية من الضحايا. Kristina Drumeller) (Bentley, 2015) (2004 لهوضوع شبه الإعتذار، كأحد الطروحات الناججة والفاعلة لكثير من المواقع، المكن من خلالها بناء صورة طبيعية عن الدولة أو المنظمة وحتى تقلل من شدة الأفكار إصلاح الصورة، ولكن ماهي السياقات وماهي إمكانية جعلهُ أكثر فاعلية في السياق المحدد؟، وقيود على وجوب أجراءات دراسات شأنها أن تضعنا في إجابات حول تساؤلات منهاهل شبه الإعتذار يمكن أن يؤدي إلى تهميش الضحايا. وجدَ الباحثان (بروك وجيلي )أن الباحثين لم يقوموا بإجراء أي تعديلات نظرية من شأنها أن تهه في إصلاح وبناء صورة المنظمة، ومع ذلك فأنهم وجدوا أن وسائل الإعلام الإجتماعية الجديدة قد تخلق صورة جيدة خصوصاً في وقت الأزمات، على العكس من وسائل الإعلام التقليدية التي تكون أقل فاعلية (Blooke Fisher, 2010). ومن أمثلة النظريات في هذا الإطار نظرية إتصال الأزمات الموقفية والتي تفترض أن المنظمات يجب أن تعطي أصحاب المصلحة أولاً، والتركيز على إهتمات المنظمة ومخاوفها لإصلاح سمعتها، فالبحوث إلى الآن تتحقق من إمكانيات نقل إطار النظرية إلى مواقع التواصل الإجتماعي، وتتسائل عن مدى إستخدام الجماهير وسائل الإعلام الإجتماعي لمواجهة وسائل المنظمة، وكذلك هو الحال لنظريتي الفوضى والتعقد فمازالت أرض بكر للأبحاث التي تجرب حول وسائل الإعلام الإجتماعي وفائدتها القصوى للمنظمات ،لذا يتحتم علينا كباحثين في مجال ادرة الازمات معرفة دور الإتصالات في الإعداد للأزمات ونقاشها لبناء الصورة في خضم الإعلام الجديد، وها هي الفرص المتاحة من إمكانيات وسائل الإعلام الجديد من بناء الصورة المرادة، وبهذا يوحد الباحثان دعوة لتجاوز دراسة كيفية إستجابة المنظمات للأزمات وبناء صورة على أساسها، والبحث في الكيفية التي يمكن لوسائل الإعلام الإجتماعية تسهيل ردود الأزمة من الجماهير لإصلاح الأخطاء وبناء الصورة الطيبة عن الدولة (Blooke Fisher, 2010). 
اعتمدت الدراسة التحليلية لخطابات خلية الازمة في محافظة واسط لمواجهة خطر انتثار فايروس كورونا التي تثكلت وفق الامر الديواني رقم (55) لسنة 2020 الصادر من الأمانة العامة لمجلس الوزراء واستتادا للادر الإداري المرقم بالعدد 24/5/1 في 2020/2/20 الصادر من محافظة واسط /مكتب المحافظ المتضمن تثكيل لجنة عليا لغرض متابعة إجراءات السيطرة على انتشار فايروس كورونا ، اذ تم تحليل (27) خطاب لخلية الازمة في محافظة واسط منذ تشكيلها لغاية 2020/3/20 ، اذ اعتمدت الدراسة لنظرية اصلاح صورة المؤسسة ومن اهم نتائج التي تم الوصول اليها وفق هذه النظرية هي : اولاً:محور استراتيجيات اصلاح الصورة في خطابات خلية الازمة : تظهر نتائج الدراسة أن استراتيجيات اصلاح الصورة لادارة العلاقات العامة اثثاء الازمات اذ كثفت النتائج ان استراتيجية الاطارات التصحيحية حلت بالمرتبة الاولى بواقع (29\% ) فيما احتلت استراتيجية الانكار المتعدد في المرتبة الثانية بواقع (26\%) كما احتلت استراتيجية التهرب من المسؤولية بالمرتبة الثالثة بواقع (20\%) وحلت استراتيجية تخفيف شناعة الحدث بالمرتبة الرابعة بواقع (15\%) وجاءت إستراتيجية الاعتراف بالذنب بالمرتبة الخامسة والاخيرة بواقع (10\%) . نظر جدول رقم

$$
\text { نتائج نظرية اصلاح الصورة في الازمات (خطابات إصلاح الصورة ) : }
$$

وفق نتائج استمارة (تحليل المضمون ) لخطابات وقرارات خلية الازمة في محافظة واسط

(جدول رقم (1) يبين استراتيجية اصلاح الصورة في خطابات خلية ازمة محافظة واسط )

\begin{tabular}{|c|c|c|}
\hline النسبة & التكرارات & 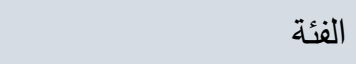 \\
\hline$\% 29$ & 29 & الإطارات التصحيحية \\
\hline$\% 26$ & 26 & الانكار المتعدد \\
\hline$\% 20$ & 20 & التهرب من المسؤولية \\
\hline$\% 15$ & 15 & تخفيف شناعة الحدث \\
\hline$\% 10$ & 10 & الاعتراف بالذنب والاعتذار \\
\hline$\% 100$ & 100 & المجموع \\
\hline
\end{tabular}

لذلك ان محور استراتيجية اصلاح الصورة ضمن خطابات خلية الازمة في محافظة واسط كان بصورة عامة يمتاز في تفاوت النتائج المستخلصة لتحليل تلك الخطابات ،اذ ظهرت اعتماد محور الإطارات التصحيحة بشكل اكبر مقارنتة ببقية المحاور •

ثانياً: محور استراتيجية اعادة الوضع والاطارات التصحيحية: تظهر نتائج الدراسة أن استراتيجيات اصلاح الصورة لادارة العلاقات العامة اثثاء الازمات اذ كثفت النتائج ان تكتيك عدم استعادة الوضع حل بالمرتبة الاولى بواقع (12\% ) فيما احتل 
تكتيك اصلاح الضرر في المرتبة الثانية بواقع (9\%) كما احتل تكتيك اصلاح الصورة او عرضي بالمرتبة الثالثة بواقع (5\%) وحل تكتيك تعويض الضحايا بالمرتبة الرابعة بواقع ( 3 \% ). نظر جدول رقم (2)..

(جدول رقم (2) يبين استراتيجية اعادة الوضع والاطارات التصحيحية لخلية الازمة في محافظة واسط)

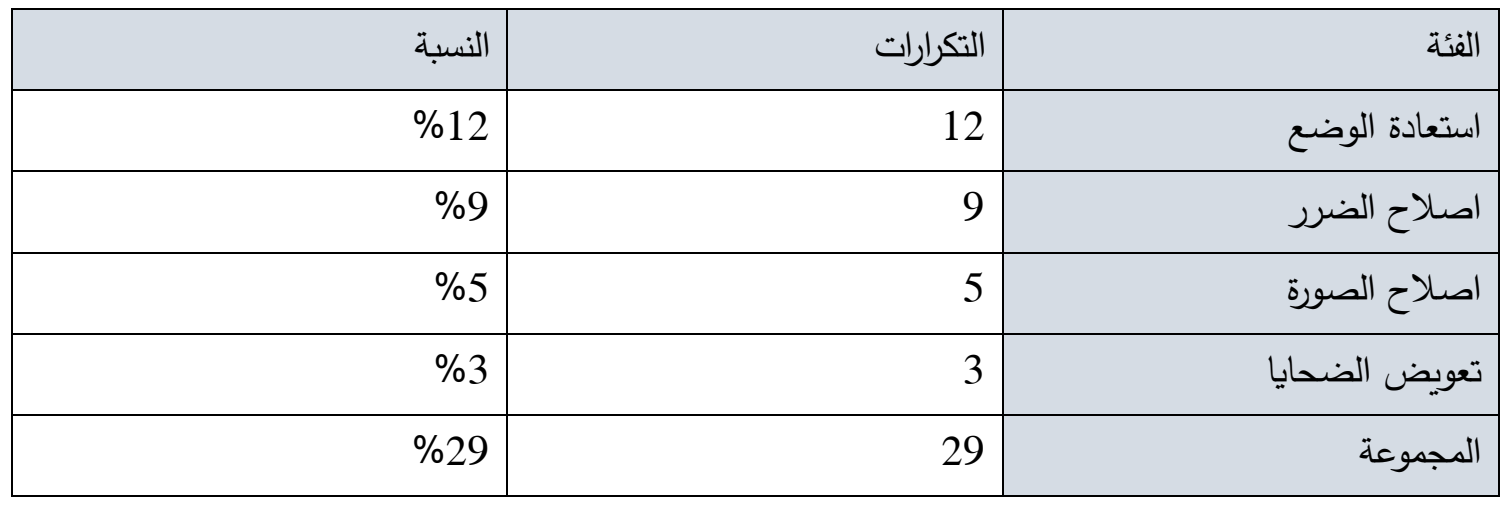

ثالثا: محور استراتيجية الانكار المتعمد : تظهر نتائج الدراسة ان الانكار المتعد ضمن استراتيجية اصلاح صورة المنظمة لادارة العلاقات العامة ان محور تحويل اللوم احتل بالمرتبة الأولى بواقع (17\%) ومحور الانكار البسيط احتل بالمرتبة الثانية بواقع (9\%) .نظر جدول رقم (3)...

(جدول رقم (3) يبين الانكار المتعد اثناء خطابات خلية الازمة في محافظة واسط )

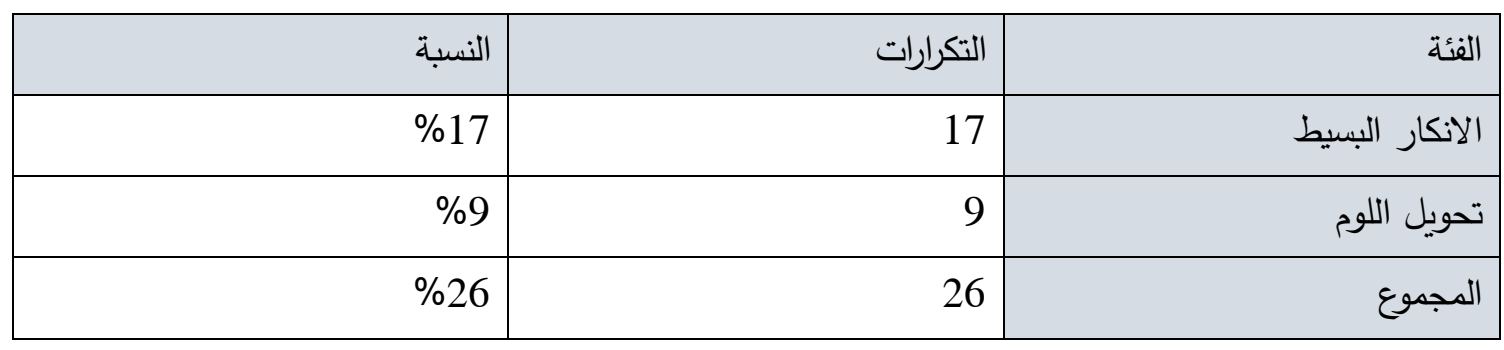

رابعآ:محور استراتيجية التهرب من لمسؤولية: تظهر نتائج الدراسة أن استراتيجيات اصلاح الصورة لادارة العلاقات العامة اثناء الازمات اذ كثفت النتائج ان تكتيك عدم امكانية المعالجة حلت بالمرتبة الاولى بواقع (7\% ) فيما احتل تكتيك وجود النوايا الحسنة في المرتبة الثانية بواقع (5\%) كما احتل تكتيك حادث طارئ او عرضي بالمرتبة الثالثة بواقع (5\%) وحل تكتيك التهرب بحجة استغزاز بالمرتبة الرابعة بواقع ( 3 \% ). نظر جدول رقم (4).. 
(جدول رقم (4) يبين استراتيجية التهرب من المسؤولية لخلية الازمة في محافظة واسط )

\begin{tabular}{|c|c|c|}
\hline النسبة & التكرارات & الفئة \\
\hline$\% 7$ & 7 & عدم إمكانية المعالجة \\
\hline$\% 5$ & 5 & وجود النوايا الحسنة \\
\hline$\% 5$ & 5 & حادث طارئ او عرضي \\
\hline$\% 3$ & 3 & التهرب بحجة الاستغزاز \\
\hline$\% 20$ & 20 & المجموع \\
\hline
\end{tabular}

خامسآ : محور استراتيجية تخفيف شناعة العدث : تظهر نتائج الدراسة أن استراتيجيات اصلاح الصورة لادارة العلاقات العامة

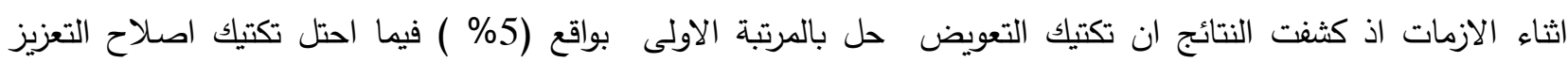
والتفاخر في المرتبة الثانية بواقع (4\%) كما احتل تكتيك التفوق على الحدث بالمرتبة الثالثة بواقع (3\%) وحل تكتيك الهجوم المضاد بالمرتبة الرابعة بواقع ( 2 \% ) فيما لم يسجل أي تكرار تكتيك التفاضل . نظر جدول رقم (5)..

(جدول رقم (5) يبين استراتيجية تخفيف شناعة الحدث المعتمدة لخطابات خلية الازمة في محافظة واسط )

\begin{tabular}{|c|c|c|}
\hline النسبة & التكرارات & الفئة \\
\hline$\% 5$ & 5 & تكتيك التعويض \\
\hline$\% 4$ & 4 & اصلاح التعزيز والتفاخر \\
\hline$\% 3$ & 3 & التفوق على الحدث \\
\hline$\% 2$ & 2 & الهجوم المضاد \\
\hline$\% 1$ & 1 & التفاضل \\
\hline$\% 15$ & 15 & المجموع \\
\hline
\end{tabular}

ساداً : محور الإعتراف بالذنب( الإعتذار): تظهر نتائج الدراسة أن استراتيجيات اصلاح الصورة لادارة العلاقات العامة اثناء

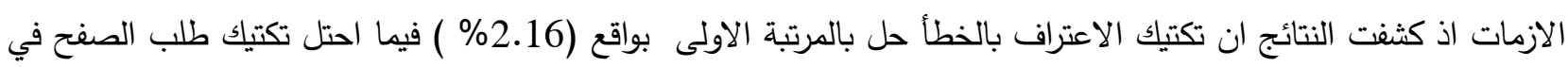
المرتبة الثانية بواقع (1.8\%) . نظر جدول رقم (6).. 
(جدول رقم (6) يبين استراتيجية الاعتراف بالخطأ اثناء خطابات خلية الازمة في محافظة واسط )

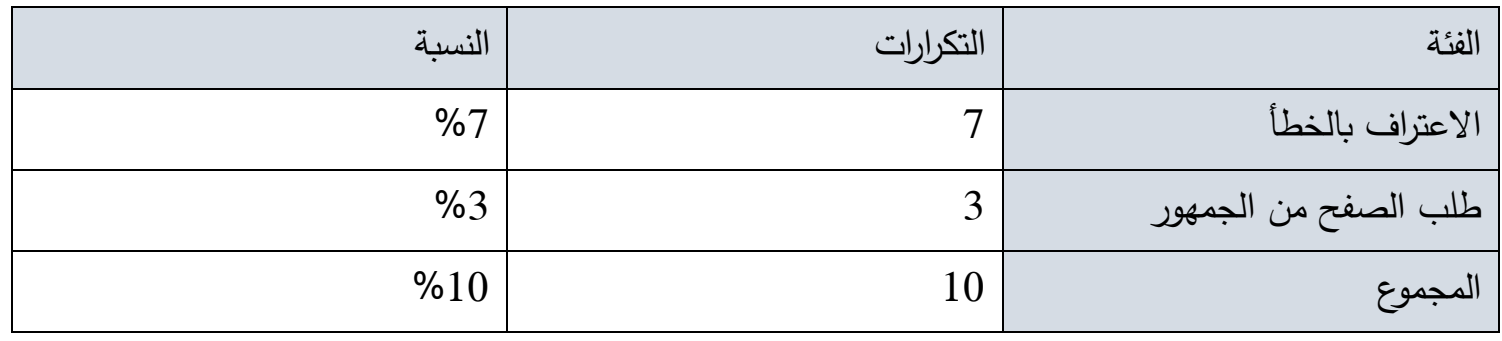

\section{سابعا : محور نتائج تحليل الوسائل الاتصالية لكلأنشطة الإعلامية لخلية الازمة في محافظة واسط :} تبين نتائج التحليل لمحور الأنشطة الاتصالية التي اعتمدتها خلية الازمة في محافظة واسط اثناء ادارتها للازمة الصحية في تعاملها مع انتثار وباء فايروس كورونا خلال مدى البحث والتحليل اذ، أوضحت النتائج باعتماد إدارة خلية الازمة على مواقع التواصل الاجتماعي بنسبة (25) من اصل ( 27) خطاب خلال تلك الفترة وهذا مايمثل نسبة ( 93\%) من اصل (100\%) وكان اعتماد خلية الازمة على الوسائل الإعلامية خلال ادارتها للازمة الصحية في محافظة واسط نسبة (2) من اصل (27) كان ذلك خلال تغطية الوسائل الإعلامية المحلية للمراسلين في محافظة واسط وتم نقلها ضمن القنوات الإعلامية التلفزيونية وهذا مايثل نسبتها (7\%) نظر جدول رقم (7)..

جدول رقم (7) يبين مدى اعتماد الأنشطة الاتصالية لخلية الازمة في محافظة واسط على الوسائل الاتصالية المتاحة

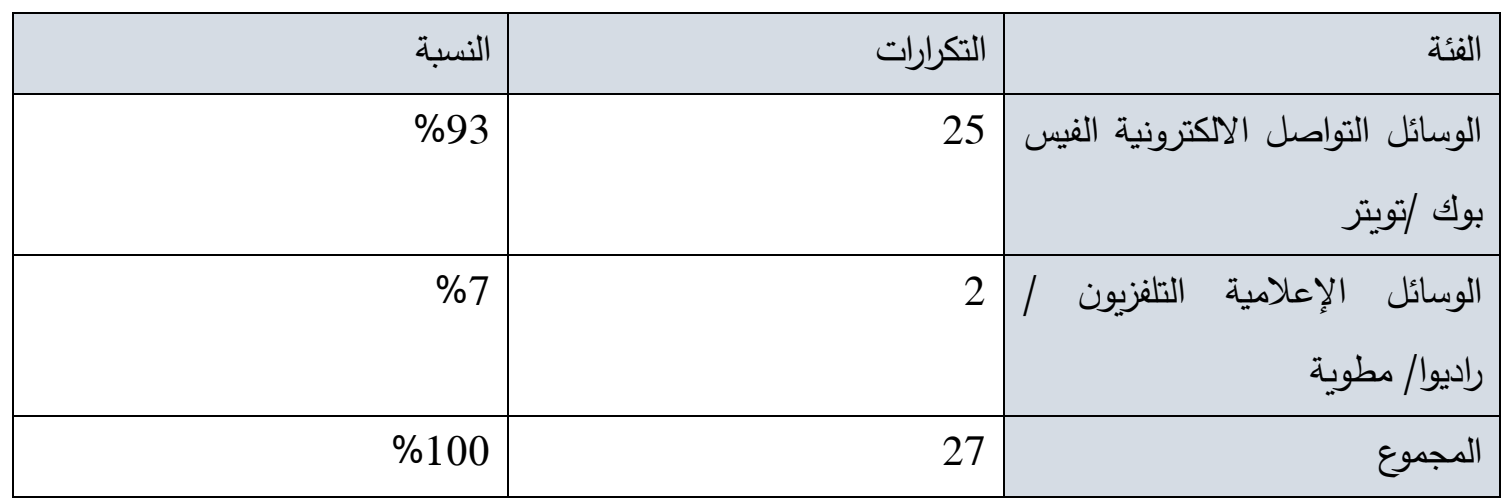

النتائج والمناقشة :

تمثل الأزمة نقطة تحول من مجموعة من الأحداث التي تؤدي إلى موقف تكون فيه الحاجة إلى العمل يشعر جميع المشاركين بحماس شديد ، ويزداد الثعور بالإحاح ، وهي حقيقة تثير التوتر والقلق أيضًا يقلل إلى حد كبير من السيطرة على التأثير والآثار ، وعادة ما تكون المعلومات المتاحة غير كافية تؤدي حقيقة لتضخيم ضغط الوقت على المشاركين وتهدد مصداقية 
لأنه يعكس جميع الجوانب المذكورة سابقا.

معظم خبراء العلاقات العامة توافق على أن أي أزمة ، في تطورها ، تتطوي على مرحلة أولية ، تحدث خلالها بعض إثارات التحذير ، المرحلة المناسبة ، والتي خلالها يجب على المنظمة التعامل مع الوضع ومرحلة ما بعد الأزمة ، خلالها تقوم المنظمة بتحليل تأثير الأزمة على الجمهور والتدابير التي يجب اتخاذها . لذلك تم الوصول الى نتائج البحث وهي :

1- تطابق محاور النظرية التي تبناها الباحث في تحليل خطابات خلية الازمة في محافظة واسط والذي توصل من خلالها الى :-

أ- عامل ظرف واحد ، أو حدث مثير ، والذي يفرض على المنظمة للتعامل مع حقائق معينة ، والتي كان حتى تلك

النقطة يمكن تجنبها

ب- عامل طارئ ، يتميز غير لائق في إدارة الموقف الناتج عن هذا الحدث . . ت- عامل هيكلي ، والذي يستمد من عدم وجود بعض هياكل لـنع الأزمات أو إدارتها . كما تسهم التصريحات المتناقضة من المسؤولين الحكومين وسوء التواصل مع الجمهور المتأثر أمثلة سلبية لإدارة حالات الأزمات، وعلى ادارة العلاقات العامة عن تحديد المخالفات المحتملة، يضاف الى ذلك عدم وجود إجراءات فورية لإدارة الأزمة ، من خطة عمل ملموسة يسلط الضوء على الإدارة السيئة التي قد تمكن بسهولة جدا ، وفقا لمبدأ كرة الثلج ، والانتقال من حدث غير سعيد مأساوي إلى مأساة . 2- لم تحاول خلية الازمة في محافظة واسط الاستفادة من الخبرات العلمية والأكاديمية والخبرات السابقة في إدارة الازمات المماثلة او المشابهة لازمة فايروس كورونا وخاصة ان القائمين على إدارة تلك الخلية كانت في موضع تتفيذ الأوامر الصادرة عن خلية الازمة المركزية وهذا الامر له طابع سلبي من حيث عدم تقدير الأوضاع الاجتماعية والاقتصادية في محافظة واسط بشكل متزن بين المحافظة على أرواح المواطنين وتطبيق إجراءات الحظر وبين توفير متتطلبات البنى التحتية للمواطنين

3- اعتماد خلية الازمة بصورة مكثفة على الأنشطة التقليدية من خلال الكتب الرسمية دون إقامة حملات العلاقات العامة بهذا الصدد مع عدم تكثيف جهودها الإعلامية بتتوع وسائلها الاتصالية اذ اقتصرت على مواقع التواصل الاجتماعي بايصال تلك الرسائل للجمهور وقد يكون هذا بفعل الوضع الطارئ الذي تمر به المحافظة نتيجة انتشار وباء فايروس كورونا في هذه المرحلة يتحتم علينا جميعاً اضفاء الطابع المؤسسي على العلاقات العامة كالإدارة الاستراتيجية وتحويلها الى وظيفة التجسير بدلاً من ممارستها الثائعة كدالة رمزية أو مؤقتة .

الاستنتاجات :

نظرا لتبني نظرية إصلاح الصورة في الازمات في العلاقات العامة وتطبيق تلك النظرية بصورة مباشرة لادارة خلية الازمة في محافظة واسط اثناء انتشار وباء فايروس كورنا يمكننا الاستتتاج في ضل هذه الإدارة مايلي : 
1- ان سمة فايروس كورنا كان سريعاً من حيث الانتثار في العالم وخصوصا في الدول المجاورة مما خلق حالة من الفوضى في بعض القرارات غير المدروسة مثل السماحات والاستثناءات التي تخللت بذخول اشخاص حاملين للفايروس وهذا جعل من خلية الازمة تكتسب المهارة والخبرة اثناء انتشار الفايروس مما ولد تلك الإدارة غير الائقة في إدارة الازمة

2- لم تكن إدارة خلية الازمة في محافظة واسط على اطلاع للخبرات العالمية بصورة فعلية او لديها ممارسات بهذا الصدد

مع منظمات محلية او عالمية في إدارة الازمات الصحية مما تطلب الاستعانة بالخبرات المتوفرة في المحافظة . 3- ان وسائل الاتصال بين إدارة خلية الازمة والجمهور كانت اعتمدت بشكل كبير على مواقع التواصل الاجتماعي ، ولم تكن هناك وسائل اتصال ذات فاعلية وتماس مابشر غير تلك المواقع •

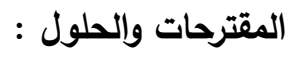

1. إضفاء الطابع المؤسسي على ادارة العلاقات العامة بمثابة انعكاس لتأثير العلاقات العامة على عمليات اتخاذ القرارات الاستراتيجية والتتظيمية ، على سبيل المثال ، أن يصبح مديرو إدارات العلاقات العامة أعضاء في مجالس الإدارة في المنظمات . 2. ضرورة " إضفاء الثرعية " على ممارسة العلاقات العامة في اغلب المؤسسات و تمكين ممارسي العلاقات العامة من لعب الأدوار الاستراتيجية ؛ يمكن التقليل من الثكوك حول "شرعية" ممارسة العلاقات العامة ؛ 3. على ممارسي العلاقات العامة تجنب الدخول مع الجماهير في لعبة "ثد الحبل" الذي يقاتل من أجل الموارد اللازمة • لإنجاز وظائفهم

4. أن تمارس العلاقات العامة بطريقة أكثر تماسكا واتساقا و مقبولة بثكل عام في الممارسة لتكون خطوة حاسمة نحو الاعتراف بها كمهنة، والمساهمة في تحقيق اهدافها 5. أن اسلوب التقييم عامل رئيسي لأنه يوضح فعالية العلاقات العامة والمساهمة التي يقدمها لتحقيق الأهداف التظيمية، وهذا بدوره قد يساعد الإدارة العليا على رؤية القيمة الحقيقية للعلاقات العامة وقبول استراتيجيتها. 6. تبني أدوار العلاقات العامة في قيادة جهود المسؤولية الاجتماعية للشركات يسهم في تحصيل على تقدير عالي "في جميع أنحاء المنظمة للعلاقات العامة ليتم دمجها في الرؤية الاستراتيجية الثاملة للمنظمة . 7. انثاء خلية لادارة الازمات في كل منظمة قبل وقوع الازمات لتكون بمثابة المجس للاشعار بوقوع الازمات ويكون احد اركانها الناطق الاعلامي 8. دعم خططها المستقبلية بأقامة برامج متخصصة ضمن هذه الخطط حول الشروع بحملات التوعية الصحية مع انثاء قنوات اتصال مع كافة الوسائل الاعلام المتاحة في المحافظة وبتنسيق معها ، هذا بالإضافة الى انشاء وسائل اتصال قادرة على الوصول للجمهور اثثاء حدوث الازمات الصحية او غيرها بما يضمن استمرارية تلك القنوات الاتصالية . 
Anna Oksiutycza, M. P. (2011). , Public relations practice in private sector companies in $\quad .1$ Gabon,. Public Public Relations Review, p. p275.

Benait, W. L. (1997). public relations, review, pp. 23- 24. .2

Benait, W. L. (1997). ,image repair discourse and crisis communication . public relations .3 review, pp. 23-24.

Bentley, J. M. (2015). , shifting identification: atheory of apdlogies and pseudo- .4 apologies, . public relations review, pp. p: 22- 29.

Bhrney, J. R. (2002). Blowout! Firestone's image resteration compagn,. public relations .5 review, p. p380.

Blooke Fisher, j. D. (2010). Beyanb image erpair: suggestions for crisis communication .6 the eay development, . public relations review , p. 643.

Bourne, C. (2019). , The public relations profession as discursive boundary work, . 7

Public Public Relations Review, p. 20.

Dipalma, S. R. (2011, march ,). , image resforation strategics employed by press 8 seeretary Robert eibbs as president barack obama,s jop approval ratings deeline, . 14 international public relations research conference, ,holiday in university off Miami, pp. 9-

Guth, D. W. (1995). Organizational Crisis Experience and Public David W. Guth 9 Relations Roles,. Publics Journalism Public Relations Review, p. p123. Harlow, W. F. (2011). BP initial image repair strategies after the deepwater horizon spill,. .10 public relations review 37, pp. pp 80-93. Joseph R. Bhrney and all, B. (2002). Firestone's image resteration compagn . public .11 relations review, p. p380.

Kristina Drumeller, W. L. (2004). , Vss Greeneville collides with Japans Ehime mam: .12 cultural issues in image repair discourse . , public relations review ,, pp. pp 177-185. 
L.Benoit, W. (2006). image repair in president bush,s apnl2004 news conference. public .13 relation review, pp. $137-143$.

Maria E. Len. Ries, W. L. (2008). ,s image rapair stratagies determined deniar and .14 jifferentiation, . public relations review 30, p. . 96.

Natalie A. Brown, A. C. (, 2013). Sports fans as crisis communicators on social media .15 websites, ,. Public Relations Review, p. 76.

Natalie A. Brown, A. C. (, 2013,). Sports fans as crisis communicators on social media .16 websites, . Public Relations Review, p. p76.

Peggy Simcic Brønn And Erik L. Olson. ( 1999,). Mapping the Strategic Thinking of .17 Public Relations Managers in a Crisis Situation: An Illustrative Example Using Conjoint Analysis, . Public Relations Review,, p. p355.

Petrovici, A. (2014). PR in Crisis Situations. A Case Study, - . Social and Behavioral .18 Sciences 149 ., p. p716.

Rhee1, B. B. (2014). Exploring Negative Dimensions of Organization-Public .19 Relationships (NOPR) in Public Relations ,. Publics Journalism \& Mass Communication Quarterly,, p. p662.

Sherry Jean Holladay, W. (2013). Timothy Coombs ,Public relations literacy: Developing .20 critical consumers of public relations. Publics Public Relations Inquir, p. p126.

Willam, B. (1997). image repair discourse and crisis communication. public relations, .21 review,, pp. 23-24.

William, K. A. (1994). Briggs Integrating Public Relations and Legal Responses During a .22 Crisis:. Public Relations Review, p. p445.

Second: Arab and Iraqi sources.

Ahmed Al Qasimi and Salim Al-Jouri. (2019 P. 21. The impact of social media sites on .23 the community security of the Gulfcountries. Germany: The Democratic Center for Strategic and Economic Studies. 
Intisar Ibrahim Abdul Razzaq, Safad Hussam Al-Samuk. (2011 P. 27. New media / .24 electronic version. Baghdad: University Printing and Publishing House.

Samir Mohammed Hussein. (1999). Media research studies in the scientific research .25 curriculum. Cairo: The world of books.

Abdul Aziz bin Sultan al-Dwehi. (2004 P. 30). Media planning and a course in the face .26 of disasters and crises. Saudi Arabia: Nayef University of Security Sciences. Abdullah Tayeh. (2006 P. 33. Cultural media in radio and television. Palestine: Al-.27 Majid Printing and Publishing House.

Abd al-Nabi Abdullah al-Tayeb. (2016 P. 35). Management of press organizations. $\quad .28$ Amman: Al Warraq Publishing and Distribution House and Foundation. Ali ajoah. (2003 P. 24. Scientific Foundations of Public Relations (Vol. Third edition). . 29 Cairo: The world of books. Kazem Omran Musa, Safad Hussam Al-Samuk. (Secondedition 2013 p. 32). University .30 media and marketing, community access strategy. Baghdad: University Printing and Publishing House.

Dry cream. (2013 P. 130. Problems of philosophy in the digital age. Baghdad: House .31 of Books and Documents. Mohammed Al-Bawi. (2004 P. 35). Communication technologies, education series for .32 telecommunication technology. Denmark: Arab League in Denmark. Huda Abd al-Rida Al-Jumaily. $(2018$, 12 26). The concept of crisis. Retrieved from .33 The University of Babylon website: http://www.uobabylon.edu.iq/uobColeges/lecture.aspx?fid=9\&depid=3\&lcid=82355 